\title{
Les débuts de la transplantation expérimentale - Considéra- tions de Charles Bonnet (1720-1793) sur la «greffe animale»
}

Par Gerhard Rudolph

Si l'historiographie médicale a un intérêt de puiser ses thèmes dans la médecine vivante comme l'a demandé Henry E. Sigerist ${ }^{1}$, si elle doit non seulement comprendre une époque en elle-même, mais introduire la notion de valeur dans ses recherches, l'exemple de Genève pourrait offrir le meilleur argument pour cette thèse. On n'a qu'à se rendre compte de la portée des travaux et des réflexions concernant la «greffe animale», travaux et réflexions qui constituent le début de la transplantologie ${ }^{2}$.

En effet, au XVIII ${ }^{\mathrm{e}}$ siècle, des Genevois se trouvent à l'avant-garde de la recherche physiologique, Genève ayant su développer depuis plusieurs générations déjà, un climat propice aux travaux d'esprit et à la recherche scientifique ${ }^{3}$. On se souvient volontiers des éloges faites par d'Alembert (1717-1783) dans un article qu'on n'a d'ailleurs pas très aimé à l'époque ${ }^{4}$ : "Toutes les sciences et presque tous les arts ont été si bien cultivés à Genève, qu'on serait surpris de voir la liste des savants et des artistes en tout genre que cette ville a produit en deux siècles. Elle a eu même quelquefois l'avantage de posséder des étrangers célèbres, que sa situation agréable, et la liberté dont on y jouit, ont engagés à s'y retirer.»

$\mathrm{Au} \mathrm{XVIII}^{\mathrm{e}}$ siècle, grâce à «cette génération d'élite qui devait embellir l'Europe en servant sa patrie» comme disait Edouard Humbert ${ }^{5}$, l'esprit de cette cité a aussi influé sur la médecine, bien que plusieurs de ses grands savants ${ }^{6}$ ne fussent pas médecins de formation, tels que Jean Jallabert (1712-1768), Abraham Trembley (1710-1784), Charles Bonnet (1720-1793), Jean Senebier (1724-1809). Leur travaux en physique, en physiologie animale et végétale, parfois sans suite immédiate pour l'art médical invétéré, leurs assurèrent pourtant une place d'honneur parmi les médecins. Ainsi Louis Jourdan (1788-1848) a-t-il réservé une place exceptionnelle de sept pages à Charles Bonnet dans sa Biographie médicale ${ }^{7}$ et, dans l'Encyclopédie de Dechambre paraît l'article "Bonnet ${ }^{8}{ }^{8}$ sorti de la plume d'Achille Chéraud (1817-1885) en 1876, à l'année même de l'inauguration de la nouvelle Faculté de Médecine ${ }^{9}$.

Évidemment au XVIII ${ }^{\mathrm{e}}$ siècle, et même au XIX ${ }^{\mathrm{e}}$, l'aspect immunologique de la transplantation des organes est encore inconnu. Les deux ordres de recherche ne se joignent que vers 1950. Mais, l'immunologie (ou ce que deviendra le noyau de l'immunologie moderne) a de très bonne heure ses défenseurs à Genève. On pense à Théodore Tronchin (1709-1781) et son article «Inoculation», un compte 
rendu de trente ans d'expérience inséré dans l'Encyclopédie de Diderot et de d'Alembert ${ }^{10}$, et à Louis Odier (1748-1817) qui a traduit Jenner en 1798 et contribué de très bonne heure à la propagation de la vaccination ${ }^{11}$. C'est l'immunologie des maladies infectieuses (Infektimmunologie) ${ }^{12}$, mais elle prépare le vaste champ des connaissances, qui permettront un jour de dévoiler le secret des rejets.

\section{Les travaux de Trembley et de Bonnet}

Quel est le premier document de la transplantation expérimentale ? En 1744 Abraham Trembley, Membre de la « Royal Society», publie à Leyde ${ }^{13}$, immédiatement suivi d'une édition parisienne ${ }^{14}$, ses Mémoires pour servir à l'histoire d'un genre de Polypes. Peu de temps après (début 1745) paraît chez le même éditeur à Paris le Traité d'Insectologie par Charles Bonnet ${ }^{15}$. L'auteur qui a juste vingtcinq ans, est Membre de la Société Royale de Londres et, dès 1740 Correspondant de l'Académie Royale des Sciences à Paris. La seconde partie du traité de Bonnet la première traite de la preuve expérimentale de la parthénogénèse ${ }^{16}$ - contient les «observations sur quelques espèces de vers d'eau douce, qui coupés par morceaux deviennent autant d'animaux complets». ${ }^{17}$ C'est-à-dire, si on divise une Hydre ou certaines Annélides en plusieurs fragments on obtient autant d'individus que de fragments ${ }^{18}$. En outre il est possible de greffer ou de transplanter un fragment sur un autre individu avec lequel il forme ensuite une unité biologique.

Il faut croire que ces publications étaient attendues par le public instruit avec impatience, car Réaumur (1683-1757), «le prince des naturalistes» - tel est le titre d'honneur que lui donne le savant genevois Émile Guyénot ${ }^{19}$ - avait déjà communiqué à ses lecteurs quelques unes des observations étonnantes de Trembley et de Bonnet. Enrichies du commentaire de Réaumur, elles occupent une large partie de la préface du sixième tome des Mémoires pour servir à l'histoire des insectes, paru en $1742^{20}$. Certains résultats étaient même diffusés avant cette date.

«Peut-on se résoudre à croire - écrit Réaumur ${ }^{21}$ - qu'il y ait dans la nature des animaux qu'on multiplie en les hachant, pour ainsi dire par morceaux ? Que d'un animal on puisse en avoir deux complets après un temps assez court ${ }^{22}$, en les coupant en deux parties? Que si on les coupe en trois, on aura trois animaux semblables et égaux à celui qui a été divisé ? Qu'enfin il y a tel animal qui étant divisé en $8,10,20,30$ et 40 parties, est multiplié autant de fois ? Chaque huitième, chaque dixième, chaque vingtième, chaque quarantième devient un animal semblable à celui dont il a été une petite portion.» Et il poursuit : «Autrefois c'était peut-être un titre à fait pour être cru, que d'être merveilleux, mais ce qui m'a paru prouver à l'honneur de notre siècle, que généralement parlant, on est parvenu à savoir douter, c'est que quoique la découverte des insectes qu'on multi- 
plie en les coupant par morceaux, ait fait une nouvelle dont on s'est beaucoup entretenu à la Cour et à la ville, cependant je n'ai vu aucune personne qui l'ait crue sur le premier récit qu'elle en avait ouï. » ${ }^{23}$ «D'ailleurs on ne saurait trop tôt rendre très publique une découverte qui à la vérité déroute nos anciennes idées, et nous jette dans de nouveaux embarras sur la nature des animaux et sur leur conformation la plus intime, mais qui étend nos vues et peut nous en faire naître de nouvelles... Au reste ce n'est pas assez d'attester la vérité d'un fait si étrange, il est nécessaire et juste de mettre en état de le voir et revoir, ceux qu'on veut convaincre de sa réalité.»

Réaumur a tout de suite su intéresser ses autres correspondants ${ }^{24}$ : GillesAugustin Bazin (1681-1754) à Strasbourg pour les Pucerons, Jean-Etienne Guettard (1715-1786) et Charles-René Girard de Villars (1698-1769) pour les Polypes que Pierre Lyonet (1707-1789) était déjà en train d'étudier. Les expériences sont multipliées, les résultats confirmés. Réaumur lui-même reprend les expériences de Bonnet sur les vers d'eau douce (expériences que le Père Mazzoleni, 1719-1768, à Rome répète avec le même succès), et il étend le champ d'investigation sur les sangsues (cette fois sans effet) et sur les planaires qui donnent de nouveau un résultat positif.

Trembley à la Haye ${ }^{25}$, Bonnet à Genève étaient en correspondance permanente avec Réaumur. Il les conseillait, il les encourageait. Ils pouvaient s'appuyer comme disait Trembley «du grand observateur qui fait l'ornement de la France et de son siècle ${ }^{26}$ et Bonnet assure en parlant de la méthode de Réaumur : «Je l'ai suivi, pour ainsi dire, pas à pas...» et il ajoute modestement : «Dans un pays si vaste et jusqu'ici assez peu fréquenté, il n'est pas difficile de faire de nouvelles découvertes. ${ }^{27}$ Le visiteur de l'Institut de France qui aura le privilège de voir s'ouvrir devant lui le dossier des correspondants de Réaumur lira avec émotion la suite de ces fascinantes découvertes. Il ne faut pas oublier que pour Karl Ernst von Baer (1792-1876) les travaux de Trembley signifiaient le commencement d'une ère nouvelle de la physiologie ${ }^{28}$.

\section{La «classe des insectes»}

En peu de temps le Polype devint un animal à la mode, suivi de près des «insectes» de Bonnet. Ni la remarque de Buffon (1707-1788) à l'adresse de Réaumur "qu'une mouche après tout ne doit pas tenir plus de place dans la tête d'un naturaliste qu'elle ne tient dans la nature $"{ }^{29}$ ni l'ironie de Diderot (1713-1784) $)^{30}$ étaient susceptibles de changer cet intérêt.

Objet de la curiosité des peintres néerlandais du XVII ${ }^{e}$ siècle qui ont découvert leurs valeurs esthétiques - comme Jan van Kessel (1626-1679) ${ }^{31}$ ou Rahel (1665- 
1750), la fille de l'anatomiste Frederik Ruysch (1638-1731) - les insectes sont passés aussitôt dans le camp des naturalistes grâce à Jan Swammerdam (1637$1680)^{32}$. Réaumur est le premier à en étudier méthodiquement la morphologie, la physiologie, l'écologie, le comportement.

Est-ce une ère entomologique qui s'annonce ? Fait curieux, Albert de Haller (1708-1777) utilise deux ex-libris, dont l'un daté 1743: ils représentent des insectes, une chenille et un papillon, portant l'inscription «non tota perit». ${ }^{33}$

Cependant, ni l'Hydre de Trembley, ni les Vers de Bonnet sont des insectes d'après notre entendement. En fait, nous nous trouvons encore à l'époque prélinnéenne. Comme sur les tableaux de Jan van Kessel, serpents et chenilles font partie d'une même classe. «La grandeur d'un animal ne doit pas suffire pour l'ôter du nombre des insectes», affirme Réaumur ${ }^{34}$. «Une chenille n'en serait pas moins chenille, si on en trouvait de plusieurs pieds de longueur. Un Crocodile serait un furieux insecte, je n'aurais pourtant aucune peine à lui donner ce nom. Tous les reptiles appartiennent à la classe des insectes par les mêmes raisons que les Vers de terre lui appartiennent. »Certes, l'homme des expériences méprise le classificateur et ce sont souvent des biologistes ou des écrivains de second ordre qui ont le souci des systèmes, tels que François Aubert de la Chesnaye des Bois (1699-1784), dont le Système naturel du Règne animal ${ }^{35}$, répondant à un besoin général de systématisation, a connu un certain succès publicitaire.

En ce qui concerne les «insectes aquatiques» on reste, même en 1750, toujours

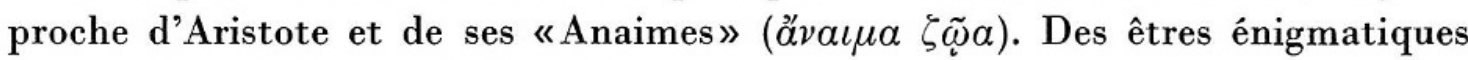
comme l'Hydre ${ }^{36}$ sont déclarés Zoophytes ou animaux-plantes, «dont la nature tient de l'animal et la figure du végétal ». ${ }^{37}$ Les fausses routes de la systématique animale offrent pourtant des aspects historiques passionnants comme l'a démontré Émile Guyénot ${ }^{38}$.

\section{La «greffe animale». La notion de régénération}

La publication quelque peu hâtive du microscopiste anglais Henry Baker (16981774) $)^{39}$ An attempt towards a natural history of the Polype, Londres 1743 - un an avant Trembley et répétant ses expériences - n'avait rien ôté à l'importance de l'œuvre du Genevois, qui restera un classique de la science expérimentale. Ceci sera aussi vrai pour ceux qui demeurent insensibles aux belles gravures de Pierre Lyonet (édition de Leyde) et qui leur préfèrent les dessins schématiques, dans quelque sens plus modernes, de Baker.

Ne connaissant pas suffisamment bien le procédé de Trembley, Baker n'a pas réussi de réunir les moitiés d'un Polype coupé en deux ou de combiner des fragments hétérogènes ${ }^{40}$. Trembley donne la déscription de ses expériences (faites en 
Novembre 1742) dans le IV ${ }^{\mathbf{e}}$ Mémoire ${ }^{41}$ : «Le hazard m'a appris a réunir des portions de Polypes : j'avais mis dans un petit verre deux morceaux de Polypes dont je m'étais servi pour faire une expérience. Les ayant examinés le lendemain, je remarquai qu'ils étaient attachés l'un à l'autre : je les coupai de nouveau, et je les rapprochai exprès; ils se réunirent encore : il n'en fallut pas davantage pour me porter à faire cette expérience avec attention. Voici comment je me prends pour réunir des portions de Polype...»

Suit une déscription minutieuse de sa technique expérimentale et des mesures à prendre pour parer aux difficultés qui pourraient survenir. Après avoir réussi ce que Alfred Giard (1846-1908) ${ }^{42}$ appellera plus tard (en 1896) la "greffe autoplastique», Trembley entreprend de réunir des portions de différents Polypes, c'est-à-dire des homoplasties et même des hétéroplasties. Ces derniers essais étaient cependant moins convaincants.

«Lorsque je fus parvenu - écrit Trembley ${ }^{43}$ - à réunir des portions de Polypes, à les greffer en quelque manière [voilà le nouveau terme] je fis d'abord part suivant ma coutume du succès de cette expérience à $M$. de Réaumur. Ce fait ne lui était pas nouveau.» Il avait même songé à réunir des Orties de mer et chargé M. de Villars de ces recherches ${ }^{44}$. Le terme de "greffe animale» qui deviendra bientôt courant a été employé, d'après mes connaissances, d'abord par Duhamel du Monceau (1700-1782) en 174645. Trembley avait déjà fait le rapprochement de ses propres observations (sur l'autogreffe) et de celles de la chirurgie. «L'expérience - dit-il ${ }^{46}$ - a appris en bien d'occasions que les parties des animaux pouvaient, lorsqu'elles se touchaient, s'attacher et se réunir aussi facilement que le font celles des plantes. C'est ce que les Médecins et les Chirurgiens ont éprouvé bien des fois à l'égard du corps humain; et c'est ce qui oblige à prendre des précautions dans le cas ou l'on veut empêcher qu'une pareille réunion se fasse.»

Les recherches de Trembley sur la régénération de l'Hydre, les investigations de Bonnet sur celle des Vers et de Bonnet et de Spallanzani (1729-1799) sur celle de la patte du Triton ${ }^{47}$, ces expériences concrètes ont «dégagé d'emblée une première notion : celle des rapports étroits entre la régénération et la greffe ». ${ }^{48}$ La série de ces travaux impressionnants débute par un travail qui montre déjà toute la maîtrise de son auteur et tout l'intérêt de ce genre de recherches, c'est le Mémoire sur la reproduction de quelques parties des écrevisses par Réaumur de $1712^{49}$.

Charles Bonnet, ayant eu la main heureuse de choisir les Vers de terre comme objets d'investigation, a vu le premier les conséquences pratiques qui résulteraient des nouvelles découvertes. Il écrit dans sa Préface de 1745: «Ces insectes étant de plus fort gros, les Médecins et les Chirurgiens pourront y étudier mieux que 
dans aucune partie de notre corps, ou de celui des animaux, tout ce qui concerne la théorie des plaies, la manière dont elles se cicatrisent et se consolident etc. Qui sait même si cela ne les conduira point à quelque découverte qui perfectionnera la Médecine et la Chirurgie ?» ${ }^{50}$

Les conséquences immédiates pour la recherche médicale ont été minimes, sinon nulles. Trembley et Bonnet avaient bientôt abandonné la recherche microscopique. Le problème de l'irritabilité occupait davantage les expérimentateurs de la seconde moitié du siècle ${ }^{51}$.

Ce n'est que vers la fin du XIX ${ }^{\mathrm{e}}$ siècle que les travaux de Trembley et de Bonnet sont repris avec la même technique (qui avait gardé toute sa fraîcheur) et aboutissants aux mêmes résultats ${ }^{52}$ : notamment par Georg Wetzel (1871-1941) à l'époque physiologiste à Breslau, par Eugen Korschelt (1858-1946), professeur de zoologie à Marbourg, et par le pathologiste Ernst Jœst (1873-1926), un disciple de Paul Ehrlich. On découvre qu'un deuxcentième du corps d'un Polype suffit pour reproduire un animal entier. Wetzel ${ }^{53}$ réunit des fragments d'une même espèce ce qu'il appelle «greffe légitime» et des espèces différentes («greffes illégitimes»). Seules les Hydres vertes et grises ne fusionnent pas. Les physiologistes Theodor Wilhelm Engelmann (1843-1909), élève de Donders, Moritz Nußbaum (1850-1915), élève de Pflüger, Hidetsurumaru Ishikawa ( ${ }^{* 1878), ~ e ́ l e ̀ v e ~ d e ~ B i e d e r-~}$ mann, et Thomas Hunt Morgan (1866-1945), directeur de l'Institut Kerckhoff à Pasadena en Californie, joignent ces initiatives par leurs propres recherches également inspirées par l'œuvre des Genevois. Une fois de plus les représentants de la médecine proprement dite admirent, mais ne se sentent pas concernés par ce qu'on appelle maintenant «Entwicklungsmechanik». ${ }^{54}$

\section{Contribution au développement de la méthode expérimentale}

Toutefois, ce qui est essentiel dans l'œuvre de Trembley et de Bonnet et ce qui sera dorénavant de la plus grande portée pour les sciences de la vie, ce n'est pas l'accumulation des données morphologiques et physiologiques, c'est le développement et l'exposition d'une méthode de recherches, initiée entre autres par Réaumur et menée à la perfection, à la dernière rigueur et clarté par les auteurs Genevois.

Quand Maupertuis (1698-1759) dans sa lettre sur le progrès des sciences (de 1752), exigea "pour faire de l'Histoire naturelle une véritable science il faudrait qu'on s'appliquât à des recherches qui nous fissent connaître non la figure particulière de tel ou tel animal, mais les procédés généraux de la nature dans sa production et sa conservation,${ }^{55}$ il n'avait qu'à tourner les yeux vers Genève et regarder la voie qui était ouverte entretemps. 
De là se dérivent, s'imposent, toutes les règles pour les investigations futures. Et c'est Bonnet qui, assisté en quelque sorte par Spallanzani, a encouragé Jean Senebier à écrire son Essai sur l'art d'observer et de faire des expériences (1775/ $1802)^{56}$, une «Somme» de la recherche qui, par ses principes mêmes, n'a rien perdu de son actualité. Car, de ces principes s'inspire en bonne partie le «raisonnement expérimental» développé au $\mathrm{XIX}^{\mathbf{e}}$ siècle. Peut-être est-il significatif que l'historiographie moderne se souvienne de ces fondements méthodiques de la recherche ${ }^{57}$, dont le contenu technique ne sera pas moins attrayant que les tendances phịlosophiques, qu'ils laissent entrevoir.

\section{Le problème des «Anciens» et des «Modernes»}

Pour comprendre le pas décisif vers 1740 dans la biologie expérimentale, il serait bon marché d'évoquer le modèle de Thomas S. Kuhn, qui, d'ailleurs, se révèle de valeur douteuse pour l'histoire des sciences ${ }^{58}$. Mais, les écrits de Trembley et de Bonnet contribuent d'une façon remarquable à une discussion qui depuis plus d'un demi-siècle (1687) ${ }^{59}$ passionne les esprits et qui ne se borne pas aux questions littéraires et artistiques, car elle affecte autant les sciences : c'est le problème des «Anciens» et des «Modernes».

Dans la Préface de 1745 Bonnet déclare : «Ce n'est que depuis le renouvellement de la Philosophie ${ }^{60}$ qu'on a commencé d'observer les insectes avec attention et par principes. Avant cette heureuse époque l'étude de la nature n'était proprement que celle des opinions de quelques philosophes. C'était moins par expériences qu'on cherchait à s'assurer des faits, que par le témoignage des Anciens. Reconnus pour les seuls dépositaires des secrets de la Nature, on les consultait comme les oracles ${ }^{61}$ et jusqu'à leurs expressions et à leurs erreurs étaient respectées.»

«Dans cet état de choses, l'Histoire Naturelle ne prenait peu ou point d'accroissement : les Naturalistes réduits à copier les Anciens, et à se recopier les uns les autres, transmettaient dans leurs écrits, avec un petit nombre de vérités, beaucoup de préjugés et d'erreurs. Enfin la nouvelle Philosophie est venue dissiper l'enchantement, et apprendre aux Physiciens à étudier la Nature dans la Nature elle-même. ${ }^{62}$

Dans les Considérations sur les corps organisés on peut lire : «...combien de merveilles inconnues au Sage et aux Anciens, que nos instruments et nos méthodes nous ont dévoilées! En rendant justice aux Anciens, il faut éviter de faire tort aux Modernes. ${ }^{63}$

Et Trembley (1744) : «Ces faits que les Philosophes n'ont pu voir, ou plutôt que le préjugé ne leur a pas permis de chercher ou de voir peut-être, quand ils étaient 
sous leurs yeux ; ces faits, dis-je, peuvent être découverts par des enfants...», et : «Il est bien vraisemblable que sans plusieurs préjugés qu'on s'est faits, l'Histoire naturelle serait plus avancée qu'elle ne l'est... ${ }^{64}$ La prise de position est nette.

\section{Bonnet Théoricien}

A Jean Rostand revient le mérite d'avoir attiré l'attention, il y a dix ans, non seulement sur l'expérimentateur, mais aussi sur le théoricien. On comprend mal le soupir de Chéraud (1876) : «mais pourquoi Ch. Bonnet n'est-il pas né avec de bons yeux ?...»

Ses œuvres "philosophiques», fruit de l'abandon forcé de la recherche expérimentale, offrent un enseignement riche pour la biologie et la biologie de la transplantation en particulier. Des Contemplations de la nature dit Jourdan ${ }^{65}$ : «cet ouvrage a plus contribué encore que ceux de Buffon à répandre le goût de l'histoire naturelle...» La médecine aurait dû en profiter elle aussi.

Les réflexions que fait Bonnet sont toujours les réflexions d'un homme de science. Ce que certifie Georges Cuvier (1769-1832) dans son éloge de 1810: «On reconnait cependant toujours dans ces écrits de son second âge, aux faits dont ils sont nourris partout, au soin avec lequel il évite de se perdre dans les systèmes fondés sur l'abus des termes abstraits, le philosophe entré dans la métaphysique par le chemin de l'observation. Le choix qu'il fit de Malebranche et de Leibnitz pour ses guides et le choix plus particulier qu'il fit entre leurs idées, rapellèrent toujours ses premiers goûts.»

«Mais ce qui le marque le mieux, ce furent des hypothèses physiques qu'il ajoutait toujours quand il avait épuisé le champ de l'observation, et par lesquelles il semblait encore vouloir offrir à l'esprit des _objets saisissables quand les sens se refusaient à lui en présenter.» Et, en pleine époque romantique, il fallait ajouter : «Ce besoin d'idées claires, presque sensibles qui constitue le veritable esprit du cartésianisme, avait été soigneusement entretenu dans l'ancienne Académie des Sciences, et Bonnet en avait été pénétré par sa correspondance avec Réaumur. ${ }^{66}$

Bonnet s'explique lui-même ${ }^{67}$ : «Je n'ai tiré des faits que les conséquences qui me semblaient en découler le plus naturellement. J'ai souhaité que mon livre fut une espèce de Logique. Je n'ai donc pas mis les Conjectures à la place des Faits ; mais j'ai fait ensorte qu'elles résultassent des Faits comme de leurs principes.» Il garde donc toujours «le fil précieux de l'expérience» et ce fut presque un titre d'honneur que d'être accusé de matérialisme par Jean-Jacques Rousseau ${ }^{68}$, «l'esprit révolté, comme disait Jean Starobinski, qui se manifeste par le refus, 
par la négativité», et «quand il parle de la nature c'est pour l'opposer à la société policée de son temps ${ }^{69}$

\section{Les germes réparateurs}

Dans les Considérations sur les Corps organisés (1762), sept chapitres sur vingt ${ }^{70}$ traitent plus ou moins explicitement des problèmes de la «greffe animale». De nombreuses remarques dans la Contemplation de la nature $(1764)^{71}$ et dans l'Essay analytique sur les Facultés de l'âme $(1760)^{72}$ complètent ces vues.

Sans insister sur le problème général de la croissance et de ses limites, sur le problème de la régénération (Bonnet tient également compte de la cicatrisation des plaies, de la formation du cal, de la force régénératrice en dépendance de l'âge du sujet), ce sont notamment deux notions qui se dégagent, qui frappent le lecteur juste à cause de leur intérêt actuel pour la biologie de la transplantation : la notion d'organisation, voire de préorganisation, et la notion de la «personne» dans la relation sujet et partie greffée.

Quant à la notion d'organisation ${ }^{73}$ Joseph Schiller a révélé l'influence du Neuchâtelois Louis Bourguet (1678-1742) sur les opinions de Bonnet ${ }^{74}$. Celui-ci, cependant, semble moins affirmatif ${ }^{75}$. En réalité, la notion d'organisation chez Bonnet ouvre des perspectives inattendues et Rostand cite à ce propos un mot remarquable de la Palingénésie (1769) : le mot de germe «désignera encore toute espèce de préformation originelle, dont un tout organique peut résulter, comme de son principe immédiat $» .^{76}$

L'existence de "germes réparateurs» ou de «germes prolifiques» disséminés dans l'organisme (car, «ce qui se régénère préexiste originairement en petit») entre dans le cadre plus général de l'hypothèse de la préformation ${ }^{77}$, qui chez Bonnet cependant garde une extrème souplesse.

Bonnet déclare ${ }^{78}$ : «...quand il s'agit de produire dans l'animal un Tout organique, la Nature paraît s'y prendre de la même manière que pour produire dans le Végétal une nouvelle branche. Elle a préformée cette branche, elle l'a renfermée en petit dans un bouton, et sa production est moins une vraie génération que le simple développement de ce qui était déjà tout formé. La nature paraît avoir de même renfermé en petit dans une espèce de bouton les parties que les insectes reproduisent à la place de celles qu'ils ont perdues...»

«Des Artères supposent des veines, les unes et les autres supposent des nerfs et bien d'autres organes. Tout cela coëxiste donc à la fois, car comment concevoir que différentes parties destinées à former un même Tout, à concourir ensemble au même but, et dont par conséquent toutes les actions sont conspirantes ou relatives, soient produites les unes après les autres par apposition...?» 
«Si l'on admet des germes particuliers pour la production des Dents, pourquoi refuserait-on d'en admettre pour la production de parties beaucoup plus composées, et dont la formation répugne encore davantage aux explications mécaniques?»

Quand Eugen Korschelt dans son traité Regeneration und Transplantation parle de «facteurs régulateurs internes», il reprend l'ideé de Bonnet ${ }^{79}$. L'étude, dit-il notamment, de la régénération des Vers de terre a montré à Bonnet que ces animaux sont susceptibles de former une tête ou une queue nouvelles. Il admettait donc l'existence de substances organisatrices («Bildungsstoffe») qui seraient transportées semblables à des courants de sève («nach Art von Saftströmen») jusqu'à l'endroit où elles déclencheraient la formation nouvelle, qui se réalise selon un plan ou un programme préexistant à cet endroit: dans la cas des Vers de terre une formation de tête dans la partie antérieure, une formation de queue dans la partie postérieure. On pense aux travaux de Hans Spemann (1869-1941, prix Nobel 1935): réalisation d'une structure par les forces inductrices de la région avoisinante («Formbildungsfähigkeit, angeregt durch Induktion vom Nachbarteil»), pour laquelle seraient responsables certaines protéines d'un poids moléculaire relativement petit (séquences de 20 à 40 acides aminés).

\section{Le problème de la «personne»}

En 1936 Maxim Bing, neurologue à Budapest, a publié un article sur «la personne biologique» dans lequel il déplore que les biologistes aient longtemps négligé un sujet comme l'individu, l'unité biologique, la personne ${ }^{80}$. Le nom de Bonnet n'est pas mentionné. Et pourtant Pierre Flourens (1794-1867), un des grands physiologistes du XIX ${ }^{\mathrm{e}}$ siècle (qui n'y figure pas non plus), avait déjà attiré l'attention sur ce point. Il écrit dans son Ontologie naturelle (1861) : «La physiologie proprement dite est une étude analytique et expérimentale... L'étude des êtres nous donne une autre science. Nous ne décomposons plus les êtres : nous les étudierons en eux-mêmes, chacun ayant son individualité, son unité propre. Je divise donc la physiologie en deux branches : la physiologie des fonctions et la physiologie des êtres... Ce mot être, dans le sens ou je m'en sers ici est un mot nouveau. On le trouve cependant un peu employé par Bonnet, qui écrivait dans la seconde moitié du dernier siècle... ${ }^{81}$

En réalité Bonnet a soigneusement étudié la question de la «personne biologique», le destin de l'être, du «Moi» surtout dans les expériences de transplantation ou de réunion, de fusion de deux individus auparavant distinctes. Il a nettement posé la question de la volonté ou de l'âme sécable.

Il traite le problème dans son Essai analytique sur les Facultés de l'âme (1760): «Dans le corps de diverses espèces d'animaux comme dans celui des plantes les 
parties essentielles à la vie sont organisées et distribuées de façon, que lorsqu'on coupe l'animal ou la plante par morceaux, chaque morceau conserve une vie qui lui est propre et reproduit toutes les parties qui lui manquaient pour être un Tout semblable à celui qu'il composait auparavant.» Et : «Que devient donc le Moi ou la Personnalité dans un animal dont il semble que nous puissions à notre gré multiplier le Moi en le coupant par morceaux ?» ${ }^{82}$

La réponse (sans insister sur les arguments ${ }^{83}$ ) : «Toutes les facultés corporelles et sensitives dans un organisme non développé ne sont... que de simples puissances.» "Ainsi il n'y a point de Personnalité dans les portions de l'animal qui n'ont point encore commencé à se compléter.»

Ou, plus explicite encore, à l'egard des Polypes greffés ${ }^{84}$ : «Quand on greffe la tête d'un Polype sur le tronçon d'un autre Polype, il est bien clair que la Personnalité ne change pas, puisque cette opération n'intéresse point le cerveau.»

«Quand on met bout à bout plusieurs portions de Polypes, elles se greffent les unes aux autres, et ne forment ensuite qu'un seul animal. La tête qui se développe dans la première portion devient le siège d'une nouvelle Personnalité.»

«Je ne sais pas ce qui arrive au cerveau de deux Polypes que l'on insère l'un dans l'autre, et dont les têtes se greffent. Mais je conçois qu'il peut survenir l'une ou l'autre de trois choses :

$1^{\circ}$ ou les deux cerveaux subsistent sans altération, et alors il y a deux personnalités distinctes,

$2^{\circ}$ ou l'un des cerveaux s'oblitère par la pression de l'autre, et alors il n'y a qu'une seule personnalité,

$3^{\circ}$ ou les deux cerveaux sont détruits, et alors il se forme une nouvelle personnalité par le développement d'un autre cerveau.»

\section{La réception des travaux des biologistes Genevois}

La question de la réception, du rayonnement des travaux de Trembley et de Bonnet, y inclus les ouvrages philosophiques, a été effleurée à plusieurs reprises. Le retentissement au XVIII ${ }^{\mathrm{e}}$ siècle a été extraordinaire ${ }^{85}$, aussi dans les milieus littéraires ${ }^{86}$. Le public médical fut informé en détail par le huitième tome des Elementa Physiologiae (1765) et le grave Haller ne cache pas son enthousiasme pour les découvertes des Genevois : "Miracula ista Abrahamus Trembley auxit...» et «Carolus Bonnet, et verax et solers auctor, animalis per mediam divisi quamque dimidiam partem, etiam decies dissectam et in frustrilla redactam, novum caput restaurare... ${ }^{87}$

Deux faits méritent d'être retenus : le physicien et philosophe allemand Georg Christoph Lichtenberg (1742-1799), utilisant le Polype d'eau douce, a répété avec 
succès les expériences de Trembley sur la greffe animale. Il a été applaudi par Blumenbach (1752-1840), anatomiste à Gœttingue, et par Gœze (1731-1793), traducteur de Bonnet et expérimentateur très habile. Les exploits de Lichtenberg ont été publiés sous forme de Lettre adressée au naturaliste Georg Forster (17541794) ${ }^{88}$.

Les travaux de Bonnet sur les vers on été repris par le médecin danois Otto Friderich Müller (1730-1784). Il parle de ses expériences dans un livre charmant ${ }^{89}$ ou il présente, gravé par lui-même, sur la page du titre les Oligochètes sous forme de ravissantes Naĩades et autres déesses grecques. Le laurier qui décorait jadis Réaumur est passé sous sa plume au Genevois. A son tour Bonnet est devenu «l'ornement de notre $\left[\mathrm{XVIII}^{\mathrm{e}}\right]$ siècle».

Un siècle presque après la mort de Bonnet, en 1881, paraît l'important rapport du physiologiste de Kiel, Victor Hensen (1835-1924), sur la "Reproduction». ${ }^{90}$ Il forme un volume entier du Traité de Physiologie de Ludimar Hermann. Ce minutieux travail fait brièvement allusion à Trembley, il se taît sur Bonnet. A tort les médecins de laboratoire avaient retiré leur confiance à un homme qu'ils regardaient comme philosophe ${ }^{91}$. En outre, à partir de la lignée SpallanzaniBaronio, retracée par Luigi Belloni ${ }^{92}$, l'histoire de la greffe animale a trouvée une suite et une autre direction avec Paul Bert (1863). Mais lui aussi en tant que physiologiste de la transplantation, s'enracine à peine plus solidement dans le milieu médical de son temps.

On peut donc conclure par la constatation toujours valable de Erwin H. Ackerknecht $^{93}$ : «Le XVIII ${ }^{e}$ siècle, le siècle des lumières a apporté à la médecine une contribution qui est aujourd'hui souvent oubliée ou méconnue.» Malgré de nombreuses études (de la part des historiens) sur les chercheurs genevois, l'intérêt médical de l'œuvre de Trembley et de Bonnet reste à méditer. La transplantologie pourrait en donner l'occasion et le lecteur qui ne s'arrête pas simplement aux faits biologiques aura l'avantage de trouver en Bonnet un interlocuteur philosophique qui l'amènera sur un terrain intéressant de la pensée biologique et dont les paroles ne s'oublient pas facilement: «Tout ce qui est doué de vie et de sensibilité a droit à la considération » ou ce temoignage d'une admirable sagesse : «J'ai toujours une place en réserve dảns mon cerveau pour les opinions contraires».

L'auteur tient à remercier les Secrétaires perpétuels de l'Académie des Sciences à Paris, Messieurs R. Courrier et P.Germain, de l'avoir autorisé de consulter dans les Archives de l'Académie les documents se rapportant à Réaumur, à Bonnet et à Trembley. - Il est redevable à la «Deutsche Forschungsgemeinschaft » d'une subvention lui permettant d'effectuer ses recherches. 


\section{Annotations}

${ }^{1}$ Henry E. Sigerist, Probleme der medizinischen Historiographie, in : Atti del VIII ${ }^{\circ}$ Congresso internazionale di Storia della medicina, Roma dal 22 al 27 settembre 1930 (ed. Pietro Capparoni), Pisa 1931, p. 119-121.

2 Dans leurs introductions historiques les auteurs traitant la biologie actuelle de la transplantation ne font guère allusion à des recherches expérimentales antérieures à Paul Bert (1833-1886). Georg L. Floersheim cependant (Transplantationsbiologie, Berlin/Heidelberg/New York 1971) remonte à Giuseppe Baronio (1759 ca.-1811). Généralement la distinction entre chirurgie réparatrice et physiologie de la transplantation est à peine faite. Même la partie historique des thèses de Paul Bert $(1863,1866)$ prête à cette confusion.

${ }^{3}$ Voir Jean Olivier, L'ancienne faculté de médecine de Genève, in: Médecine de France, $\mathrm{N}^{0} 78$ (1956), p. 14-16, 32 et 48 ; Huldrych M. Koelbing, Que devons-nous, en médecine, à la Suisse romande ?, Gesnerus 32 (1975), p. 123-127; pour les sciences en général, leurs processus évolutifs : Cléopâtre Montandon, Le développement de la science à Genève aux $X V I I I^{e}$ et $X I X^{e}$ siècles - Le cas d'une communauté scientifique, Vevey 1975.

${ }^{4}$ L'article «Genève» du tome VII de l'Encyclopédie a été réimprimé dans CEuvres complètes de d'Alembert, T. IV, Paris 1822, p. 411-421. Voir aussi «Avertissement sur la Justification de l'Article Genève de l'Encyclopédie», ibid., p. 423-425, et «Lettre à J. J. Rousseau, citoyen de Genève», ibid., p. 432-458.

${ }^{5}$ Edouard Humbert, Charles Bonnet disciple de Montesquieu, Bibliothèque universelle, LXIII $^{\mathrm{e}}$ année, nouvelle période (1858), p. 525-551.

${ }^{6}$ Cf. Cléopâtre Montandon, Sciences et Société à Genève aux $\mathrm{XVIII}^{\mathrm{e}}$ et $\mathrm{XIX}^{\mathrm{e}}$ siècles, Gesnerus 32 (1975), p. 16-34.

${ }^{7}$ Louis Jourdan, article «Bonnet» in: Biographie médicale (Panckoucke), vol. 2, Paris 1820, p. 369-375. Sur Bonnet nous avons consulté : Georges Cuvier, Éloges historiques de Charles Bonnet et Horace-Bénédict de Saussure (1810) in: Recueil des Éloges historiques lus dans les Séances publiques de l'Institut royal de France I, p. 383-430, Strasbourg et Paris 1819; André Sayous, Charles Bonnet, sa vie et ses travaux d'après une correspondance inédite, Revue des deux mondes 12 (1855), p. 49-81; Raymond Savioz, La philosophie de Charles Bonnet de Genève, Paris 1948; Jean Rostand, Un grand biologiste, Charles Bonnet, expérimentateur et théoricien, Palais de la Découverte (D 107), Paris 1966 ; et les documents communiqués par Raymond Savioz (ed.), Mémoires autobiographiques de Charles Bonnet de Genève, Paris 1948, et Carlo Castellani (ed.), Lettres à M. l'abbé Spallanzani de Charles Bonnet, Milano 1971.

${ }^{8}$ Amédée Dechambre (ed.), Dictionnaire des Sciences médicales, $\mathrm{I}^{\mathrm{re}}$ série, T.10, Paris 1876, p. 68-70.

${ }^{9}$ G. Bickel, La Faculté de médecine de Genève, Médecine de France, $\mathrm{N}^{0} 78$ (1956), p. 3-6.

10 [Théodore] Tronchin, Article «Inoculation», in : Encyclopédie ou Dictionnaire raisonné des Sciences, des Arts et des Métiers par une Société de Gens de lettres, T.VIII ${ }^{\mathrm{e}}$, Neufchastel 1765, p. 755-771.

11 Georges de Morsier, La vie et l'œuvre de Louis Odier, docteur et professeur en médecine (1748-1817), Gesnerus 32 (1975), p. 248-270.

12 Cf. François Dagognet, L'immunité, histoire et méthode, Palais de la Découverte (D 94), Paris 1964. 
${ }^{13}$ Abraham Trembley, Mémoires pour servir à l'histoire d'un genre de Polypes d'eau douce, à bras en forme de cornes, Leyde 1744.

${ }^{14}$ Chez Durand dans la rue Saint Jacques, à S. Landry et au Griffon, 2 vols., Paris 1744. L'édition de Leyde contient les remarquables illustrations dessinées et gravées par le naturaliste hollandais Pierre Lyonet (1707-1789), un «observateur pénétrant et expérimenté» qui, à cette époque, «mania pour la première fois de sa vie, la pointe et le burin» (Trembley, Préface). L'édition de Paris ne reproduit que quelques unes des planches hors-texte de Lyonet; elle supprime les dessins de Cornelis Pronk (1691-1759) et les autres gravures exécutées par Jacob van der Schley (1715-1779).

15 Charles Bonnet, Traité d'Insectologie, première et seconde parties, Paris 1745. «Il aurait paru en 1744, si le libraire m'avait tenu parole», Mémoires autobiographiques, l.c. p. 82. Jean-Baptiste Haussard (1679 ?-1749), le graveur de Réaumur, a executé les planches pour les deux parties d'après les dessins de Bonnet.

${ }^{16}$ Hubert Erhard, Die Entdeckung der Parthenogenesis durch Charles Bonnet, Gesnerus 3 (1946), p. 15-27.

${ }^{17}$ Lumbriculus. Cf. Jean Rostand, l.c. (annotation 7) p. 12.

${ }^{18}$ Maurice Doyon en traitant du pouvoir de reproduction des animaux inférieurs peu différenciés cite Réaumur, Baker et «Trembley principalement» sans parler des travaux de Bonnet; J.-P.Morat et Maurice Doyon, Traité de Physiologie, T.V, Paris 1918, p.470s.

${ }^{19}$ Emile Guyénot, Les sciences de la vie au XVII ${ }^{e}$ et XVIII ${ }^{e}$ siècles, L'idée d'évolution, Paris 1957, p. 195.

${ }^{20}$ [René-Antoine Ferchault] de Réaumur, Mémoires pour servir à l'histoire des Insectes, 6 volumes, Paris 1734-1742; Travaux de Bonnet et de Trembley voir Préface du Tome VI, p. XLV-LXXX.

${ }^{21}$ Réaumur, ibid., p. XLIXs.

${ }^{22}$ "La reproduction [après coupe transversale] se fait plus ou moins vite, suivant qu'il fait plus ou moins chaud. J'ai vu dans le fort de l'Été, des secondes parties dont les bras ont commencé à pousser au bout de vingt-quatre heures; et qui, en deux jours, ont été en état de manger ; et dans un temps froid, j'en ai vu dont la tête n'a été formée qu'en quinze ou vingt jours.» Trembley, l.c. IV. Mém., p.165. Et : «il ne faut communément qu'une heure, et même moins pour que la moitié d'un polype coupé suivant sa longueur, prenne la forme d'un polype parfait», ibid., p.187.

${ }^{23}$ Sur la discussion de «la plus étrange et la plus embarrassante nouveauté qui se soit jamais offerte à ceux qui étudient la nature» (Réaumur à Bonnet, 30 nov. 1741) cf. Jacques Roger, Les sciences de la vie dans la pensée française du XVIII ${ }^{e}$ siècle, Paris 1963, p. 394s. et passim.

${ }^{24}$ Voir aussi Jean Torlais, Réaumur d'après des documents inédits, éd. revue et augmentée, Paris 1961.

${ }^{25}$ La première communication date du décembre 1740 (Réaumur, l.c. p.LIII.

${ }^{26}$ Guyénot, l.c. (annotation 19) p.195-196.

27 Bonnet, Insectologie, Préface, p.IX.

${ }^{28}$ Eduard Fueter, Correspondance inédite entre Réaumur et Abraham Trembley (analyse), Gesnerus 1 (1944), p.110-112, p.110.

${ }^{29}$ Cité d'après Jean Torlais, Réaumur philosophe, in: Pierre-P.Grassé (éd.), La vie et l'œuvre de Réaumur (1683-1757), Paris 1962, p.160. 
30 «Aux grands génies les grands objets, les petits objets aux petits génies» (Pensées sur l'interprétation de la nature), voir Torlais, ibid., p.161.

${ }^{31}$ Deux tableaux du maître d'Anvers étaient exposés récemment à Paris (VIII ${ }^{\mathrm{e}}$ Biennale des Antiquaires, octobre 1976) par la Galerie David M. Koetser (Zurich) : «Insectes» 1657 (le tableau représente le nom de l'artiste composé de serpents et de chenilles) et «Fleurs et Insectes» 1659. Le champ des peintres d'insectes était certainement plus étendu. Nous ignorons combien les autres membres de la famille Ruysch p.ex. ou Willem van Aelst, le maître de Rahel, y ont contribué.

${ }^{32}$ Historia insectorum generalis ofte Algemeen verhandeling van de bloedelose dierkens, Utrecht 1669 ; éditions en langue française Utrecht 1682, 1685, en latin (Leyde) 1685, Utrecht 1693 ; Bybel der natuur en néerlandais et en latin, avec la vie de l'auteur par Hermann Boerhaave (1668-1738), Leyde 1737-1738. Voir aussi A.Schierbeek, Jan Swammerdam (1637-1680). His life and his works. Amsterdam 1967.

${ }^{33}$ Edouard Bonnet, Les ex-libris de Albert de Haller, Bulletin de la Société française d'Histoire de la Médecine 13 (1914), p.197-201.

${ }^{34}$ Insectes seraient donc «tous les animaux que leurs formes ne nous permettent pas de placer dans la classe des quadrupèdes ordinaires, dans celle des oiseaux et dans celle des poissons », Réaumur, Mémoires etc. I (1734), Préface, p. 58.

35 [François-Alexandre Aubert de la Chesnaye des Bois], Système naturel du Règne animal par classes, familles ou ordres, genres et espèces [etc.], 2 vols., Paris 1754. L'auteur suit l'ordre de Linné (1707-1778) en faisant la distinction entre la classe des Insectes et celle des Vers, que ce dernier divise en Reptiles, Zoophytes, Testacés et en Lithophytes. Voir T. 2, p. $253 \mathrm{~s}$.

${ }^{33}$ Voir Aubert de la Chesnaye, 1.c. T.2, p. 263-264. L'auteur donne une liste des chercheurs qui ont travaillé sur l'Hydre. - Le nom de Polype pour l'Hydre d'eau douce a été proposé par Réaumur en 1742 (Préface T.VI, p.LIV). Cf. Jacques-Christophe Valmont de Bomare (1731-1807), Dictionnaire raisonné d'Histoire naturelle, $3^{\text {e }}$ éd., Lyon 1776, Article «Polype», T.VII, p. 256-271.

${ }^{37}$ Dictionnaire des Sciences naturelles contenant l'histoire des Animaux, des Végétaux, des Minéraux etc. (faussement attribué à Buffon), 2 vols., Amsterdam 1781, II, p. 493-494 (Zoophytologie). Cf. Trembley, l.c. IV. Mem., p.521 s.

${ }^{38}$ Guyénot, l.c. Chapitres IV (Les systèmes zoologiques au XVIII ${ }^{\mathrm{e}}$ siècle...) et V (La classification des Invertébrés...), p. 61-93.

${ }^{39}$ Henry Baker (F.R.S.), An attempt towards a natural history of the Polype in a letter to Martin Folkes, president of the Royal Society, London 1743. - Une traduction française a été publiée par Pierre Demours (1702-1795), médecin de la Faculté de Paris et oculiste du Roi, sous le titre Essai sur l'histoire naturelle du Polype, insecte, Paris 1744.

${ }^{40}$ Baker, l.c. Experiment XIX : An attempt to make the divided Parts of different Polypes unite, p.175-177.

${ }^{41}$ Trembley, l.c. T. II, p. 285 s.

42 Alfred Giard, Y a-t-il antagonisme entre la «greffe » et la «régénération » ? C.R. Soc. Biol. (Paris), 15 févr. 1896, réimpr. dans Euvres diverses I, Biologie générale, Paris 1911, p. 471-475.

${ }^{43}$ Trembley, l.c. T. II, p. 294.

${ }^{44}$ Lettre de Réaumur du 14 Déc. 1742 citée par Trembley, ibid., p. 294. 
45 [Henri-Louis] Du Hamel [Du Monceau], Recherches sur la réunion des plaies des arbres, sur la façon dont la greffe s'unit au sujet sur lequel on l'applique, sur la réunion des plaies des animaux, et sur quelques exemples de greffes appliquées sur des animaux, Hist. de l'Acad. Royale des Sciences, Année 1746, Amsterdam 1755, p.475-534; p. 516.

${ }^{46}$ Trembley, l.c. T. II, p. 308.

47 [Lazzaro] Spallanzani, Programme ou Précis d'un ouvrage sur les Reproductions animales par Mr. l'Abbé Spallanzani..., traduit de l'italien [Prodromo sopra le riproduzioni animali, Modena 1768] par B***** de la Sabionne, Genève 1768. - Charles Bonnet, Expériences sur la régénération de la Tête du Limaçon terrestre, Journal de Physique 1777, p. 246-283; 3 Mémoires sur la reproduction des membres de la Salamandre aquatique, Journal de Physique 1778, p. 284-358. - Cf. Carlo Castellani (ed.), Lettres de Bonnet à Spallanzani (Annot. 7), passim.

${ }^{48}$ Raoul-Michel May, La greffe (L'avenir de la Science, $\mathrm{N}^{\circ} 33$, Coll. dir. par Jean Rostand), Paris 1952, p.11.

${ }^{49}$ Réaumur, Sur les diverses Reproductions qui se font dans les Écrevisses, les Omars, les Crabes etc. et entres autres sur celles de leurs Jambes et de leurs Écailles, Hist. de l' Acad. Royale des Sciences, Année 1712, Paris 1777, p.45-47 (Histoire), p.295-321 (Mémoire).

${ }^{50}$ Bonnet, l.c. p. XXVI-XXVII.

${ }^{51}$ Cf. Gerhard Rudolph, Hallers Lehre von der Irritabilität und Sensibilität, in : Karl Eduard Rothschuh (ed.), Von Boerhaave bis Berger - Die Entwicklung der kontinentalen Physiologie im 18. und 19. Jahrhundert unter besonderer Berücksichtigung der Neurophysiologie, Stuttgart 1964, p.14-34 ; - L'irritabilité Hallérienne, Point de départ de la Neurophysiologie, in: Actualités Neurophysiologiques, Paris 1967, p. 295-319.

${ }^{52}$ L'histoire récente de la transplantation expérimentale est traitée dans les études de Eugen Korschelt, Regeneration und Transplantation, Jena 1907; «Regeneration» in: Handwörterbuch der Naturwissenschaften 8 (1913), p.160-199, à compléter par l'article de Dietrich Barfurth "Transplantation», ibid., 10 (1915), p.1-18; et Eugen Korschelt, Regeneration und Transplantation, 3 vols., Berlin 1927-1931.

${ }^{53}$ Georg Wetzel, Transplantationsversuche mit Hydra, Arch. mikroskop. Anat. 52 (1898), p. 70-96.

${ }^{54}$ Curt Herbst, Entwicklungsmechanik oder Entwicklungsphysiologie der Tiere, in : Handwörterbuch der Naturwissenschaften 3 (1913), p.545s.

55 [Pierre-Louis Moreau de] Maupertuis, Euvres, nouvelle édition, 4 vols., Lyon 1768, T. II, p. 418 ; voir aussi Émile Callot, Maupertuis le savant et le philosophe, Paris 1964.

${ }^{56}$ Jean Senebier, L'art d'observer, Genève 1775, et : Essai sur l'art d'observer et de faire des expériences, 3 tomes, Genève 1802. Des deux ouvrages se trouvant à la Bibliothèque nationale de Paris, le second se présente dans une magnifique reliure aux armes de la Bibliothèque impériale sur les plats que l'on pourrait regarder, si ce n'était pas un procédé courant, comme marque de l'estime portée à un ouvrage exceptionnel. «En lisant l'Essai analytique sur les facultés de l'âme de Bonnet, je saisis ses idées grandes et profondes sur l'art d'observer, cette lecture tourna souvent mes idées sur cette belle matière; je me déterminai à m'en occuper davantage pour rechercher les bases de cet art » (Senebier, T. 3, p. 8). Sur l'influence de Spallanzani et de Pierre Prévost (1751-1839) voir ibid., p.14.

${ }^{57}$ Cf. Jacques Marx, L'art d'observer au XVIII ${ }^{\mathrm{e}}$ siècle: Jean Senebier et Charles Bonnet, Janus 61 (1974), p.201-220. 
${ }^{58}$ Cf. les critiques formulées récemment par Fritz Krafft à l'exemple de Copernic: «Progressus retrogradis. Die 'Copernicanische Wende' als Ergebnis absoluter Paradigmatreue», XIII. Symp. Société d'Histoire des Sciences, Münster 1975.

${ }^{59}$ [Bernard le Bovier de] Fontenelle (1657-1757), Digression des anciens et des modernes, Paris 1687; cf. Gerhard Rudolph, Wissenschaft und Wissenschaftsverständnis in der französischen Aufklärung, Sudhoffs Archiv 61 (1977), p. 19-37.

${ }^{60} \mathrm{Cf}$. le rôle que d'Alembert attribue à Francis Bacon (Discours préliminaire de l'Encyclopédie, 1751).

${ }^{61}$ Le nom de Fontenelle semble s'imposer.

${ }^{62}$ Bonnet, Insectologie, Préface, p. I-II.

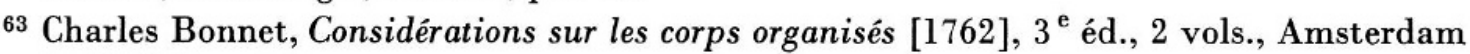
1776, T.I, p.181.

${ }^{64}$ Trembley, l.c. IV. Mém., p. 224-226.

${ }^{65}$ Jourdan, l.c. (annotation 7) p. 375.

${ }^{66}$ Cuvier, l.c. (annotation 7) p. 396.

${ }^{67}$ Bonnet, Corps organisés, T.I, p.XIV et XXII.

${ }^{68}$ Jean-Jacques Rousseau, Confessions, Partie II, Livre XII, in : Euvres complètes, 8 tomes, Paris 1864, VI, p.168: «... ledit Bonnet, quoique matérialiste ne laisse pas d'être d'une orthodoxie très intolérante sitôt qu'il s'agit de moi.»

${ }^{69}$ Jean Starobinski, Rousseau et Buffon, in : Jean-Jacques Rousseau et son œuvre, Colloque de Paris (1962), p.135-146.

${ }^{70} \mathrm{Y}$ compris les «considérations sur la formation des monstres».

${ }^{71}$ Charles Bonnet, Contemplation de la nature [1764], $2^{\mathrm{e}}$ ed., 2 vols., Amsterdam 1769. L'ouvrage est dédié à Haller, Duhamel Du Monceau, Trembley et Allamand.

${ }^{72}$ Charles Bonnet, Essay analytique sur les Facultés de l'âme [1760], seconde édition, 2 tomes, Copenhague et Genève 1769.

${ }^{73}$ Cf. aussi [Georges Leclerc de] Buffon (éd. Jean Piveteau, Corpus-général des Philosophes Français, XLI, 1) Paris 1954, p.248 A, 245 B, 246 A.

${ }^{74}$ Joseph Schiller, La notion d'organisation dans l'œuvre de Louis Bourguet (1678-1742), Gesnerus 32 (1975), p.87-97.

${ }^{75}$ Bonnet, Corps organisés, Chap. XII, 210, p.189: Observation sur le sentiment de Mr. Bourguet et de quelques autres Auteurs touchant la prétendue Organisation des Sels, des Cristaux, des Pierres.

${ }^{76}$ Rostand, l.c. (annotation 7) p. 21.

77 Jacques Roger, l.c. La préexistence des germes, p. 334-384, Les difficultés de la préexistence, p.385-439; Joseph Needham, A history of Embryology, 2nd ed., Cambridge 1959; Jacques Marx, La préformation du germe dans la philosophie biologique du XVIII ${ }^{\mathrm{e}}$ siècle, Tijdschrift voor de Studie van de Verlichting 1 (1973), p.397-428.

${ }^{78}$ Bonnet, Corps organisés, T. II, p. 22 et 25.

${ }^{79}$ Korschelt, l.c. (annotation 52) I, p. 669.

${ }^{80}$ Maxim Bing, Die «biologische Person», Ciba Zeitschrift (Bâle) 4 (1936), p.1362-1368.

${ }^{81}$ Pierre Flourens, Ontologie naturelle ou Étude philosophique des Êtres, Paris 1861, p. 2 et 3.

82 Bonnet, Essai analytique, Paragr. 755, p.247. Cf. Joseph Schiller, Queries, answers and unsolved problems in eighteenth century biology, History of Science 12 (1974), p.184-199, p.189s. 
${ }^{83}$ Bonnet, Essai analytique, Paragr. 756-769, p. 247-253.

${ }^{84}$ Bonnet, Corps organisés, T. II, p. 72.

${ }^{85}$ La preuve est le nombre des savants répétant les expériences sur l'Hydre ou sur les Vers je ne mentionne que Roesel von Rosenhof (1705-1759) devenu très connu par ses Insektenbelustigungen - et la qualité des traducteurs et commentateurs. Ainsi Titius (Johann Daniel Tietz, 1729-1796, professeur de Mathématique) a traduit la Contemplation de la nature (plusieurs éditions: 1766, 1772, 1774, 1783); Johann August Ephraim Goeze (voir Jourdan, l.c. 4, p.475-478) est le traducteur de l'Insectologie de Bonnet (Halle 1773, 1774).

${ }^{86}$ On trouve de nombreuses remarques dans la correspondance de Grimm. Plusieurs passages chez Goethe, Lessing et autres prouvent que l'intérêt restait longtemps éveillé.

${ }^{87}$ Albert de Haller, Elementa Physiologiae corporis humani, Ed. secunda, T.VIII (Fetus hominisque vita), Lausanne 1778, p.164-167, voir notamment la description brillante et concise des expériences sur le Polype, p.165.

${ }^{88}$ Georg Christoph Lichtenberg, Physikalische und mathematische Schriften (ed. J.L. Lichtenberg et F.Kries), Göttingen 1804, vol. 4, p.231-247 [première publication: Hanovre 1775].

${ }^{89}$ Otto Friderich Müller, Von Würmern des süßen und salzigen Wassers, Copenhague 1771, p. 33, 34, passim. Son ouvrage Vermium terrestrium et fluviatilium seu Animalium infusoriorum, Helminthicorum et Testaceorum, non marinorum, succincta historia (Copenhague et Leipzig 1773) est dédié à Haller, Linné et Bonnet.

${ }^{90}$ Victor Hensen, Die Physiologie der Zeugung, in: Ludimar Hermann, Handbuch der Physiologie, T.VI, 2.Theil, Leipzig 1881.

${ }^{91}$ Le physiologiste Albrecht Bethe a très bien expliqué les hésitations du chercheur devant la spéculation philosophique (néovitaliste) dans sa Allgemeine Physiologie, Berlin/Göttingen/Heidelberg 1952, p.139; Jean Théodoridès se place sous un angle de vue comparable en rapprochant Bonnet de la « Naturphilosophie» du XIX ${ }^{\mathrm{e}}$ siècle, Histoire de la Biologie, Paris 1965, p. 55.

${ }^{92}$ Luigi Belloni, Dalle riproduzioni animali di L. Spallanzani agli innesti animali di G. Baronio, Physis 3 (1961), p.37-48.

${ }^{93}$ Erwin H.Ackerknecht, Grands médecins suisses 1500-1900, Palais de la Découverte (D 109), Paris 1966, p.10. 


\section{Summary}

The physiology of transplantation begins about 1740 with the experimental work done by the Genevan scientists A.Trembley and Ch. Bonnet. In a narrow collaboration with Réaumur, they were able to demonstrate the connection between regeneration (Réaumur 1712) and transplantation. The authors clearly recognized the importance of this new field of research for medical and surgical applications. Their rigorous experimental method, a model for biological investigations, stimulated the Genevan J. Senebier to search for the principles and to codify $(1775 / 1802)$ the rules of observation and experimentation in natural science. The biophilosophical writings of Bonnet (since 1760) are of particular interest for modern transplantology, for they discuss in an explicit manner a) the question of the organization of living material and its intrinsic regenerating forces, b) the problem of the biological "person", which arises when an individual or parts of him are grafted upon or fused with other individuals. The intellectual attitude of Bonnet and Trembley furnishes a striking example of a major problem of the early Enlightenment persisting in the middle of the 18 th century, namely, the opposition between the "Ancient" and the "Modern", i.e., between dogmatists and experimentalists.

Prof. Dr. med. Gerhard Rudolph

Institut für Geschichte der Medizin und Pharmazie

Brunswiker Straße 2 a

D-23 Kiel 